 \\ TATRA \\ MOUNTaiNS \\ Mathematical Publications
}

\section{ON THE SEPARATELY OPEN TOPOLOGY}

\author{
Zbigniew Piotrowski - Robert W. Vallin - Eric Wingler
}

\begin{abstract}
We consider the relationship between separately continuous functions and separately open sets, and we study the properties of the separately open topology on $\mathbb{R}^{2}$ and on $\mathbb{Q}^{2}$. We show that $\mathbb{R}^{2}$ with this topology (denoted $\mathbb{R} \otimes \mathbb{R}$ ) is completely and strongly Hausdorff and that $\mathbb{Q} \otimes \mathbb{Q}$ is normal but not a $p$-space. In addition, we show that each point of $\mathbb{Q} \otimes \mathbb{Q}$ has an uncountable neighborhood base.
\end{abstract}

\section{Introduction}

This paper deals with two topologies on the space $\mathbb{R}^{2}$, the usual Euclidean topology and the separately open (or plus) topology. In this paper we will compare and contrast these topologies and the $G_{\delta}$ sets formed by each.

Let $f$ be a function from $\mathbb{R}^{2}$ into $\mathbb{R}$. We say that $f$ is continuous with respect to $x$ (with respect to $y$ ) if the restricted function $f_{y}(x)=f(x, y)$, where $y$ is fixed $\left(f_{x}(y)=f(x, y)\right.$, where $x$ is fixed) is a continuous function from $\mathbb{R}$ into $\mathbb{R}$. If $f$ is continuous with respect to both $x$ and $y$, then $f$ is called a separately continuous function. The canonical example of a function that is separately continuous at a point where it is not continuous, is

$$
f(x, y)=\left\{\begin{array}{cl}
\frac{2 x y}{x^{2}+y^{2}}, & (x, y) \neq(0,0), \\
0, & (x, y)=(0,0) .
\end{array}\right.
$$

Since $f$ is not continuous at $(0,0)$, we know that there exist open intervals $I=(-a, a)$ such that $f^{-1}(I)$ is not an open Euclidean set in the plane. It is natural to ask what such a set $f^{-1}(I)$ looks like. The answer is a separately open set containing the origin.

Definition 1. The $\varepsilon$-plus at $(a, b)$ of radius $\varepsilon>0$ is

$$
P_{\varepsilon}(a, b)=\left\{(x, b) \in \mathbb{R}^{2}:|x-a|<\varepsilon\right\} \cup\left\{(a, y) \in \mathbb{R}^{2}:|y-b|<\varepsilon\right\} .
$$

(Note: We shall use $B_{\varepsilon}(a, b)$ to denote a Euclidean open ball about $(a, b)$.)

2000 Mathematics Subject Classification: Primary 05C38, 15A15; Secondary 05A15, $15 \mathrm{~A} 18$.

Keywords: separately open topology, separate continuity. 


\section{ZBIGNIEW PIOTROWSKI — ROBERT W. VALLIN — ERIC WINGLER}

More generally, if $X$ and $Y$ are topological spaces, $(p, q) \in X \times Y$, and $U$ and $V$ are open neighborhoods of $p$ and $q$, respectively, we define the $(U, V)$-plus at $(p, q)$ by

$$
P_{U, V}(p, q)=\{(x, q): x \in U\} \cup\{(p, y): y \in V\} .
$$

Definition 2. A set $G \subset \mathbb{R}^{2}$ is said to be separately open if for each point $(a, b) \in G$ there exists $\varepsilon>0$ such that $P_{\varepsilon}(a, b) \subset G$.

In general, the separately open topology is formed as follows: Let $X_{1}, X_{2}, \ldots$ $\ldots, X_{n}$ be a finite collection of topological spaces and let $X=\prod_{i=1}^{n} X_{i}$. We say that $S \subset X$ is separately open provided that for each $x=\left(x_{1}, x_{2}, \ldots, x_{n}\right) \in S$ and each $i=1,2, \ldots, n$ there is a neighborhood $N_{i}$ of $x_{i}$ in $X_{i}$ such that $\prod_{i=1}^{n} A_{i} \subset S$ where $A_{j}=\left\{x_{j}\right\}$ when $j \neq i$ and $A_{i}=N_{i}$. For more information, see [6] and [7].

\section{Structure of separately open sets}

It is obvious that Euclidean open sets are separately open. The following example shows that the converse is not true.

ExAmple 1. The Maltese Cross

$$
A=\{(0,0)\} \cup\left\{(x, y) \in \mathbb{R}^{2}:|y|>|3 x|\right\} \cup\left\{(x, y) \in \mathbb{R}^{2}:|y|<\left|\frac{x}{3}\right|\right\}
$$

is a separately open, but not Euclidean open set.

The Maltese Cross has only one point $(0,0)$ where it is not open in the usual sense; that is, it is the union of an open set with a singleton. Obviously, one can quickly come up with a set with an infinite number of such points. For example, let

$$
A_{(0,0)}=A \cap\left[\left(-\frac{1}{2}, \frac{1}{2}\right) \times\left(-\frac{1}{2}, \frac{1}{2}\right)\right],
$$

and let $A_{(i, j)}=(i, j)+A_{(0,0)}$ for each $(i, j) \in \mathbb{Z}^{2}$. Then $\cup\left\{A_{(i, j)}:(i, j) \in \mathbb{Z}^{2}\right\}$ is separately open, but each point $(i, j) \in \mathbb{Z}^{2}$ lies outside of the (Euclidean) interior.

EXAMPLE 2. Another example of a separately open set that is not Euclidean open was given by $\mathrm{P}$ o p v a s s i le v [12]. Remove any circle from the plane letting one point $P$ of this circle remain. The remaining set is separately open, but $P$ is not in the (Euclidean) interior. 


\section{ON THE SEPARATELY OPEN TOPOLOGY}

These routine examples motivate us to ask the following question: Where can these points of "essential" separate openness occur; that is, can a nonempty separately open set be constructed in a way different from adding points to an existing nonempty open set?

The answer to this question is yes. We mention here a few ways to show this. One of the easiest examples to construct is the following: Let $\alpha$ and $\beta$ be real numbers such that

$$
\alpha^{2}+\beta^{2}=1 \text { and } \frac{\alpha}{\beta} \notin \mathbb{Q},
$$

and let $f$ be the rotation defined by

$$
f(x, y)=(\alpha x+\beta y,-\beta x+\alpha y) .
$$

Then it can be easily seen that the set $G=f\left(\mathbb{Q}^{2}\right)$ has the property that every horizontal or vertical line intersects it in at most one point. Hence $\mathbb{R}^{2} \backslash G$ is separately open. Since $G$ is dense in $\mathbb{R}^{2}$ under the usual topology, $\mathbb{R}^{2} \backslash G$ cannot be obtained by adding points to an existing nonempty open set.

The following is a construction that can be generalized to other topological spaces. In the unit square $I \times I$, where $I=(0,1)$, pick a countable base $\mathcal{B}=$ $\left\{B_{1}, B_{2}, \ldots\right\}$. Using induction, we shall first construct a dense countable set $D$ that has at most one point in common with every horizontal and every vertical segment. (Such a set $D$ is called a dense thin subset of $I \times I$, see [11.) First, choose an arbitrary point $\left(x_{1}, y_{1}\right)$ of $B_{1}$. Suppose that for some natural number $n$ we have already chosen $\left(x_{1}, y_{1}\right),\left(x_{2}, y_{2}\right), \ldots,\left(x_{n}, y_{n}\right)$ such that $\left(x_{i}, y_{i}\right) \in B_{i}$ and if $i \neq j$, then $x_{i} \neq x_{j}$ and $y_{i} \neq y_{j}$. Since every set in $\mathcal{B}$ is of cardinality $\mathfrak{c}$, by the Pigeonhole Principle we can pick $\left(x_{n+1}, y_{n+1}\right) \in B_{n+1}$ such that $x_{n+1} \neq x_{i}$ and $y_{n+1} \neq y_{i}$ for $i=1,2, \ldots, n$. Let $D=\left\{\left(x_{n}, y_{n}\right): n \in \mathbb{N}\right\}$. By construction, the set $D$ is countable and dense. Now, let $G=(I \times I) \backslash D$. It is easy to see that $G$ is separately open. The above construction can be generalized to fairly general topological spaces, e.g., both spaces in the product being Baire spaces having countable $\pi$-weight. (For results on thin and very thin dense sets, see [16], 13], and [5].)

Finally, $\mathrm{H}$ art and $\mathrm{K}$ u n e n [6, Remark 2.2] give the following example. Let $f: \mathbb{R} \rightarrow \mathbb{R}$ be a $1-1$ function whose graph $\Gamma$ is dense in the plane. Then $\mathbb{R}^{2} \backslash \Gamma$ is separately open. However, since $\Gamma$ is dense in the plane, $\mathbb{R}^{2} \backslash \Gamma$ has an empty interior, so it cannot be derived by adding points to a nonempty open set.

Example 3. The Maltese Cross $A$ is a $G_{\delta}$ set in the Euclidean topology. If we let $A_{n}=A \cup B_{1 / n}(0,0)$, then each $A_{n}$ is Euclidean open and $\cap A_{n}=A$.

TheOREM 1. If $C$ is a separately open subset of $\mathbb{R}^{2}$ and is Euclidean open at all points except those in a set $E \subset C$ that is a $G_{\delta}$ set in the Euclidean topology, then $C$ is a $G_{\delta}$ set in the Euclidean topology. 


\section{ZBIGNIEW PIOTROWSKI — ROBERT W. VALLIN — ERIC WINGLER}

Proof. Since $E$ is a $G_{\delta}$ set, there is a countable collection of Euclidean open sets $U_{n}$ such that $E=\cap U_{n}$. The set $C_{n}=C \cup U_{n}$ is Euclidean open for each $n$ and $C=\cap C_{n}$.

Question. The set $E$ can be finite or in some cases countably infinite, but how far can we extend this exceptional set? Will any countable set do? What about a nowhere dense set?

It is not the case, though, that every separately open set is a Euclidean $G_{\delta}$ one.

ExAmple 4. Let $S=\{(x, x): x \in \mathbb{R} \backslash \mathbb{Q}\}$ and let $G=\mathbb{R}^{2} \backslash S$. Then each $x$-section and each $y$-section is open in $\mathbb{R}$ so $G$ is separately open. However, $G$ is not a Euclidean $G_{\delta}$ set because if it were, then $G \cap\{(x, x): x \in \mathbb{R}\}$ would be a $G_{\delta}$ subset of the line $y=x$. This is impossible since this set is homeomorphic to $\mathbb{Q}$.

This example shows that it is not sufficient for the set $E$ in Theorem 11 to be countable. We note that in this example the set $\mathbb{R} \backslash \mathbb{Q}$ could be replaced by any other subset of $\mathbb{R}\left(G_{\delta}\right.$ or not, nor even Borel) and the resulting set $G$ would be separately open. This shows that the cardinality of the collection of separately open sets in $\mathbb{R}^{2}$ is $2^{\mathfrak{c}}$, and since the cardinality of the collection of Borel subsets of $\mathbb{R}^{2}$ is $\mathfrak{c}$, there must exist separately open sets that are not Borel sets.

An interesting fact about the usual topology on $\mathbb{R}^{2}$ is that each open set can be expressed as the inverse image of an open set in $\mathbb{R}$ under some continuous function. In particular, if $G \subset \mathbb{R}^{2}$ is open and $f(x)$ is the distance from $x$ to $\mathbb{R}^{2} \backslash G$, then $f^{-1}((0, \infty))=G$.

Question. Is every separately open set in $\mathbb{R}^{2}$ the inverse image of an open set in $\mathbb{R}$ under a separately continuous function?

The answer to this question is no, as can be seen by the following cardinality argument. The cardinality of $\{G \subset \mathbb{R}: G$ is open $\}$ is $\mathfrak{c}$, and since a separately continuous function on $\mathbb{R}^{2}$ is uniquely determined by its values on a dense subset (such as $\mathbb{Q}^{2}$ ) of $\mathbb{R}^{2}$ (see [14), the cardinality of the set of separately continuous functions is $\mathfrak{c}$. Hence the cardinality of

$$
\left\{f^{-1}(G): G \text { is open in } \mathbb{R} \text { and } f: \mathbb{R}^{2} \rightarrow \mathbb{R} \text { is separately continuous }\right\}
$$

is $\mathfrak{c}$. However, the cardinality of the collection of all separately open sets in $\mathbb{R}^{2}$ is $2^{\mathfrak{c}}$. It follows that most separately open sets in $\mathbb{R}^{2}$ cannot be expressed as the inverse image of an open set in $\mathbb{R}$ under a separately continuous function. 


\section{Generalized separate oscillation}

In this section we will assume that all spaces are Hausdorff.

Let $Z$ be a topological space. A sequence $\left\{\mathcal{G}_{n}: n \in \mathbb{N}\right\}$ of open covers of $Z$ is called a development of $Z$ if for each $z \in Z$ the set $\left\{s t\left(z, \mathcal{G}_{n}\right): n \in \mathbb{N}\right\}$ is a base at $z$. A regular developable space is called a Moore space.

Further, a completely regular space $Z$ is a $p$-space if and only if there exists a sequence $\left\{\mathcal{G}_{n}: n \in \mathbb{N}\right\}$ of families of open subsets of $\beta Z$ such that

(1) each $\mathcal{G}_{n}$ covers $Z$;

(2) for each $z \in Z, \cap\left\{s t\left(z, \mathcal{G}_{n}\right): n \in \mathbb{N}\right\} \subset Z$.

The following term was introduced in [8]:

Definition 3. We say that a topological space $Z$ has the property $\left({ }^{*}\right)$ if there is a sequence $\left\{\mathcal{G}_{n}: n \in \mathbb{N}\right\}$ of open covers of $Z$ such that if $z \in G_{n} \in \mathcal{G}_{n}$ for each $n$, and if $W$ is an open set in $Z$ that contains z, then $\cap\left\{G_{j}: 1 \leq j \leq n\right\} \subset W$ for some $n$.

In the class of completely regular spaces, $p$-spaces with a $G_{\delta}$-diagonal coincide with spaces that have property $(*)$. Also, every developable space has the property $(*)$. (See [8] for additional information.)

Refining the generalized oscillation $\omega_{f}$ introduced in [8], we will now define a generalized separate oscillation $\omega_{f}^{\text {sep }}$ of $f: X \times Y \rightarrow Z$. Define the generalized separate oscillation $\omega_{f}^{\text {sep }}$ of $f$ on the $(U, V)$-plus $P=P_{U, V}(p, q)$ by

$$
\omega_{f}^{\text {sep }}(P)=\inf \left\{\frac{1}{n}: n \in \mathbb{N}, \exists G \in \mathcal{G}_{n} \text { such that } f(P) \subset G\right\} .
$$

The generalized oscillation $\omega_{f}^{\text {sep }}$ of $f$ is defined by

$$
\omega_{f}^{s e p}(p, q)=\inf \left\{\omega_{f}^{s e p}(P): P \in \mathcal{P}(p, q)\right\},
$$

where $\mathcal{P}(p, q)$ stands for the collection of all $(U, V)$-pluses at $(p, q)$.

\section{An extension theorem}

It is well-known [10, p. 422] that if $f$ is a continuous function defined on a subset $A$ of a metric space $X$ with values in a complete metric space $Y$, then there exists a continuous extension $f^{*}$ of $f$ to a $G_{\delta}$ subset $A^{*}$ of $X$. This motivates us to look for an analogous result for separately continuous functions defined on subsets of the Cartesian plane $\mathbb{R}^{2}$. To begin, let $A$ be a subset of $\mathbb{R}^{2}$, and let $f$ be a real-valued separately continuous function defined on $A$; that is, the restrictions of $f$ to each horizontal and vertical section of $A$ are continuous. 


\section{ZBIGNIEW PIOTROWSKI - ROBERT W. VALLIN - ERIC WINGLER}

Call a point $p$ a weak plus-accumulation point of $A$ if $p$ is an accumulation point of $P_{1}(p) \cap A$ in the usual topology on $\mathbb{R}^{2}$. Call a point $p=(x, y)$ a plus-accumulation point of $A$ if $p$ is an accumulation point of both $(\{x\} \times \mathbb{R}) \cap A$ and $(\mathbb{R} \times\{y\}) \cap A$ in the usual topology on $\mathbb{R}^{2}$. Let $A^{+}$denote the set $\mathrm{A}$ together with all its plus-accumulation points. For each point $p$ in $A^{+}$define $\omega^{+}(f, p)$, the separate oscillation $]^{1}$ of $f$ at $p$, to be the oscillation considered only over pluses at $p$; that is,

$$
\omega^{+}(f, p)=\lim _{r \rightarrow 0} \sup \left\{\left|f\left(q_{1}\right)-f\left(q_{2}\right)\right|: q_{1}, q_{2} \in A \cap P_{r}(p)\right\} .
$$

(Notice that if $p$ is an isolated point of $A$, in the sense that $P_{r}(p) \cap A=\{p\}$ for some $r$, then $\omega^{+}(f, p)=0$.) Let $A^{*}$ be the set of points $p$ in $A^{+}$for which $\omega^{+}(f, p)=0$. To each $p=\left(x_{0}, y_{0}\right)$ in $A^{*}$ assign the sequence $\left\{p_{n}\right\}$ in $A$ with $p_{n} \rightarrow p$ and $p_{n}=\left(x_{0}, y_{n}\right)$ or $p_{n}=\left(x_{n}, y_{0}\right)$. Since $\omega^{+}(f, p)=0$, we have

$$
\lim _{n \rightarrow \infty} \operatorname{diam}\left(f\left(\left\{p_{n}, p_{n+1}, \ldots\right\}\right)\right)=0 \text {. }
$$

So $\left\{f\left(p_{n}\right)\right\}$ is a Cauchy sequence whose limit we will denote as $f^{*}(p)$. Then $f^{*}$ is the extension of $f$ to $A^{*}$. Separate continuity follows directly from the fact that $\omega^{+}\left(f^{*}, p\right)=0$. Hence we have the following:

Theorem 2. Let $f: A \rightarrow \mathbb{R}$ be a separately continuous function where $A \subset \mathbb{R}^{2}$ and let $A^{*}$ be as defined above. If $A$ is a proper subset of $A^{*}$, then $f$ has a separately continuous extension to $A^{*}$.

Remark 1. The statement of this theorem is far weaker than we would have liked, which would have been to say that $A^{*}$ is a separately $G_{\delta}$ set (that is, the intersection of a countable collection of separately open sets). While it is true that the set of points in $A^{+}$where $\omega^{+}\left(f^{*}, p\right)=0$ is the intersection of the sets

$$
A_{n}=\left\{p \in A^{+}: \omega^{+}\left(f^{*}, p\right)<\frac{1}{n}\right\},
$$

we cannot say that the sets $A_{n}$ are separately open. For suppose $p \in A_{n}$. To show that $A_{n}$ is open, we would need to show that there is $r>0$ such that $P_{r}(p) \cap A^{+}$is contained in $A_{n}$. However, for any $r>0$ there may exist points $q$ in $P_{r}(p) \cap A^{+}$such that $\omega^{+}\left(f^{*}, q\right) \geq 1 / n$, simply because there are points from $A$ that lie on a plus centered at $q$ that do not lie on a plus centered at $p$.

Even if all of the sets $A_{n}$ were separately open, we still would not be able to say that $f$ could be extended to a separately $G_{\delta}$ set, because it is not clear that $A^{+}$is a separately $G_{\delta}$ set. While it is true that every horizontal and vertical section of a separately $G_{\delta}$ subset of $\mathbb{R}^{2}$ is a $G_{\delta}$ subset of $\mathbb{R}$, the following question

\footnotetext{
${ }^{1}$ Separate oscillation and generalized separate oscillation (mentioned in the previous section) are related. However, the former is an extended real-valued function, while the latter is bounded above by 1 .
} 


\section{ON THE SEPARATELY OPEN TOPOLOGY}

remains: If every horizontal and vertical section of a subset $A$ of $\mathbb{R}^{2}$ is a $G_{\delta}$ subset of $\mathbb{R}$, is $A$ a separately $G_{\delta}$ set?

Upon examining the proof of the preceding theorem, one might think that instead of using plus accumulation points in the definition of $A^{+}$, we could have used weak plus accumulation points instead. The following example will show that this is not always possible.

EXAMPLE 5. Let

$$
f(p)= \begin{cases}1 & \text { if } p \in \mathbb{Q} \times(\mathbb{Q} \backslash\{0\}) \\ 0 & \text { if } p \in(\mathbb{R} \backslash \mathbb{Q}) \times(\mathbb{R} \backslash \mathbb{Q}),\end{cases}
$$

and let

$$
A=[\mathbb{Q} \times(\mathbb{Q} \backslash\{0\})] \cup[(\mathbb{R} \backslash \mathbb{Q}) \times(\mathbb{R} \backslash \mathbb{Q})]
$$

Then $f$ is separately continuous on $A$, because it is constant on each horizontal and each vertical section. Each point $p=(x, 0)$ is a weak plus-accumulation point of $A$, and $\omega^{+}(p)=0$. However, $f^{*}(p)$ will be either 0 or 1 depending on whether $x$ is irrational or rational. Hence, $f^{*}$ is not separately continuous on $A^{*}$.

The next example demonstrates a limitation on the above method used to obtain an extension.

ExAmple 6 . Let $A$ be a countable dense subset of $(0,1)^{2}$ having at most one point in common with each horizontal and each vertical line. (The construction of such a set $D$ is demonstrated in the text following Example 2.) Also, let $B$ and $C$ be disjoint subsets of $A$ such that both $B$ and $C$ are dense in $A$ and $A=B \cup C$. Now, consider the following two functions:

(1) $f_{1}: A \rightarrow \mathbb{R}$, defined by $f_{1}(p)=1$ for each $p \in A$, and

(2) $f_{2}: A \rightarrow \mathbb{R}$, defined by $f_{2}(p)=1$ for each $p \in B$ and $f_{2}(p)=-1$ for each $p \in C$.

Note that the extension function $f_{1}^{*}$ is given by $f_{1}^{*}(p)=1$ for each $p \in(0,1)^{2}$, but $f_{1}^{*}$ cannot be obtained by the "sequence techniques" used above, because $A$ has no plus-accumulation points. For the same reason, our technique does not extend $f_{2}$ continuously either.

The authors are grateful to the referee for supplying the previous example.

For abstract topological spaces, a corresponding result is Theorem 1.1 of [2]. 


\section{ZBIGNIEW PIOTROWSKI — ROBERT W. VALLIN — ERIC WINGLER}

\section{Separation axioms}

In this section we will discuss which separation axioms the plus topology satisfies. To distinguish between the space $X \times Y$ with the product topology and the space $X \times Y$ with the plus topology, we will denote the latter by $X \otimes Y$.

Henriksen and Woods [7] have shown that if each of $X$ and $Y$ has a countable $\pi$-weight and $Y$ is a Baire space, then $X \otimes Y$ is not regular. A more explicit construction showing that $\mathbb{R} \otimes \mathbb{R}$ is not regular is provided by $\mathrm{Hart}$ and $\mathrm{Kun}$ en [6], where it is shown that if $D \subset \mathbb{R} \times \mathbb{R}$ is dense in the Tychonoff topology and can be viewed as the graph of a $1-1$ function that is closed and discrete in the plus topology, then the non-regularity of $\mathbb{R} \otimes \mathbb{R}$ follows from Sierpinski's theorem (see [6]), which asserts that every such separately open set is dense in the plus topology. Yet another construction showing the nonregularity of $\mathbb{R} \otimes \mathbb{R}$, based on the Baire Category theorem, was provided by Popvasilev [12].

The space $\mathbb{R} \otimes \mathbb{R}$ is clearly Hausdorff because its topology is stronger than the usual topology, which is Hausdorff. More generally, it is shown in [6] that $X \otimes Y$ is Hausdorff if and only if both $X$ and $Y$ are Hausdorff.

Similar arguments can be made for the properties Urysohn, completely Hausdorff, and strongly Hausdorff. A space $X$ is Urysohn (see [15]) if for each pair of distinct points $x$ and $y$ in $X$ there is a continuous function $f: X \rightarrow[0,1]$ such that $f(x)=0$ and $f(y)=1$. The space $\mathbb{R} \otimes \mathbb{R}$ is Urysohn because $\mathbb{R}^{2}$ is Urysohn, and a continuous function on $\mathbb{R}^{2}$ is also continuous on $\mathbb{R} \otimes \mathbb{R}$.

A space $X$ is completely Hausdorff (see [15]) if for each pair of distinct points $x$ and $y$ there exist disjoint open sets $U$ and $V$ such that $x \in U, y \in V$, and $\bar{U} \cap \bar{V}=\varnothing$. If $X$ is a Urysohn space, then it is completely Hausdorff. Hence, $\mathbb{R} \otimes \mathbb{R}$ is completely Hausdorff.

A Hausdorff space $X$ is strongly Hausdorff (see [9]) if every infinite subset of $X$ contains a sequence $\left\{x_{n}\right\}$ such that the terms $x_{n}$ have pairwise disjoint neighborhoods in X. Again, since $\mathbb{R}^{2}$ is strongly Hausdorff and the plus topology is stronger than the usual topology, $\mathbb{R} \otimes \mathbb{R}$ is strongly Hausdorff as well; that is, a collection of pairwise disjoint neighborhoods in $\mathbb{R}^{2}$ is also a collection of pairwise disjoint neighborhoods in $\mathbb{R} \otimes \mathbb{R}$.

\section{Other topological properties}

TheORem 3. A neighborhood base for a point in $\mathbb{Q} \otimes \mathbb{Q}$ must be uncountable.

P r o of. Suppose that $\left\{B_{n}\right\}$ is a countable neighborhood base of the point $(x, y)$ in $\mathbb{Q} \otimes \mathbb{Q}$. We will construct inductively an open set $G$ containing $(x, y)$ such 


\section{ON THE SEPARATELY OPEN TOPOLOGY}

that $B_{n} \not \subset G$ for each $n$. Let $\left(x_{1}, y_{1}\right)$ be a point in $B_{1} \backslash\{(x, y)\}$. Suppose that points $\left(x_{1}, y_{1}\right),\left(x_{2}, y_{2}\right), \ldots,\left(x_{n-1}, y_{n-1}\right)$ different from $(x, y)$ have been selected respectively from $B_{1}, B_{2}, \ldots, B_{n-1}$ so that no two of these points lie on the same horizontal or vertical line. Since $B_{n}$ contains a plus centered at $(x, y)$, there is a point $\left(x_{n}, y\right)$ in $B_{n}$ with $x_{n} \neq x$ such that $x_{n} \neq x_{i}$ for all $i=1,2, \ldots, n-1$. Now $B_{n}$ contains a plus centered at $\left(x_{n}, y\right)$, so there is a point $\left(x_{n}, y_{n}\right)$ in $B_{n}$ with $y_{n} \neq y$ and $y_{n} \neq y_{i}$ for all $i=1,2, \ldots, n-1$. Hence $\left(x_{n}, y_{n}\right) \in B_{n} \backslash\{(x, y)\}$ and $\left(x_{n}, y_{n}\right)$ does not lie on any horizontal or vertical line containing $\left(x_{i}, y_{i}\right)$ for any $i<n$. Now, $G=\mathbb{Q}^{2} \backslash\left\{\left(x_{n}, y_{n}\right): n \in \mathbb{N}\right\}$ is an open set and $B_{n} \not \subset G$ for each $n \in \mathbb{N}$. Hence, a neighborhood base of $(x, y)$ cannot be countable.

Remark 2. Since there are at most $\mathfrak{c}$ subsets of $\mathbb{Q}^{2}$ and a neighborhood base of $\mathbb{Q} \otimes \mathbb{Q}$ must be uncountable, under the Continuum Hypothesis there must be exactly $\mathfrak{c}$ open neighborhoods of a point.

In view of the above construction, the cardinality of the neighborhood base of $\mathbb{R} \otimes \mathbb{R}$ must be uncountable. In fact, a neighborhood base for a point in $\mathbb{R} \otimes \mathbb{R}$ must have $2^{\mathfrak{c}}$ elements. This is an immediate corollary of the following theorem (see also [18, p. 739]).

TheORem 4 ([6, Lemma 2.1, p. 105]). Suppose that $X$ and $Y$ are Hausdorff spaces, that $w(X) \leq \mathfrak{c}$, and that each non-empty open subset of $X$ has size at least $\mathfrak{c}$. Suppose that there are disjoint countable sets $D_{\alpha} \subset Y$ for $\alpha<\mathfrak{c}$ such that each $D_{\alpha}$ is dense in $Y$. Then,

$$
\chi((p, q), X \otimes Y) \geq 2^{\mathfrak{c}} \quad \text { for all } \quad(p, q) \in X \times Y .
$$

(For a discussion of the weight $w(X)$ of a topological space $X$ and the character $\chi(p, X)$ of a point in $X$, see [3, pp. 27-28].) Note that our Theorem 3 does not imply nor is implied by this result.

Remark 3. A. V. Arhangelski $\breve{1}$ [1] introduced a class of spaces, called $p$ spaces, in the following way: $X$ is called a $p$-space (cf. 4, p. 444]) if there exists a sequence $\left\{\mathcal{G}_{n}\right\}$ of open covers of $X$ satisfying the following condition: For each $x \in X$ and each $n$, if $G_{n}$ satisfies $x \in G_{n} \in \mathcal{G}_{n}$, then

(1) $\cap_{n} \bar{G}_{n}$ is compact, and

(2) $\left\{\cap_{i \leq n} \bar{G}_{i}: n \in \omega\right\}$ is an outer network for the set $\cap_{n} \bar{G}_{n}$; that is, every open set containing $\cap_{n} \bar{G}_{n}$ contains some $\cap_{i \leq n} \bar{G}_{i}$.

The class of $p$-spaces is rather large; it contains all metric spaces and all Čech-complete spaces. In the same article [1, A r hange Iskiǔ showed that if $X$ is a $p$-space, $w(X) \leq \operatorname{card}(X)$ (see [9], Remark, p. 10).

Obviously, $\operatorname{card}\left(\mathbb{Q}^{2}\right)=\omega$, but we have just shown that $w(\mathbb{Q} \otimes \mathbb{Q})$ is uncountable. This proves that $\mathbb{Q} \otimes \mathbb{Q}$ is not a $p$-space. 


\section{ZBIGNIEW PIOTROWSKI - ROBERT W. VALLIN - ERIC WINGLER}

Remark 4. It is natural to ask whether $\mathbb{Q} \otimes \mathbb{Q}$ is a regular space. In fact, it is. Recall (see [6]) that a $\sigma$-set is a separable metric space in which every $F_{\sigma}$ set is also a $G_{\delta}$ set. Since every countable metric space (in particular, $\mathbb{Q}$ is a $\sigma$-set and $\mathbb{Q}$ is a countable non-discrete metric space, it follows from $[6$, Theorem 5.5, p. 118] that $\mathbb{Q} \otimes \mathbb{Q}$ is regular.

One of the cardinals used in set theory is the cardinal $\mathfrak{p}$ (see [17, p. 115]). It is known [17, Theorem 3.1(a), p. 116] that $\mathfrak{p} \geq \omega_{1}$. It follows from [6, Corollary 5.8, p. 119] that $\mathbb{Q} \otimes \mathbb{Q}$ is normal and strongly 0-dimensional. (For a definition of strongly 0-dimensional, see [3, p. 443].) Of course, it would be nice to see an elementary proof of the normality of $\mathbb{Q} \otimes \mathbb{Q}$.

\section{REFERENCES}

[1] ARHANGEL'SKIJ̆, A. V.: On a class of spaces containing all metric spaces and all locally bicompact spaces, Sov. Math. Dokl. 4 (1963), 1051-1055.

[2] BURKE, D.-LUTZER, D.-LEVI, S.: Functional characterizations of certain p-spaces, Topology Appl. 20 (1985), 161-165.

[3] ENGELKING, R.: General Topology, in: Monografie Matematyczne 60, PWN-Polish Scientific Publishers, Warszawa, 1977.

[4] GRUEnHAGE, G.: Generalized Metric Spaces, in: Handbook of Set-Theoretic Topology (K. Kunen, J. E. Vaughan, eds.), North-Holland, Amsterdam, 1984.

[5] GRUENHAGE, G.-NATKANIEC, T.-PIOTROWSKI, Z.: On thin, very thin, and slim dense sets, Topology Appl. 154 (2007), 817-833.

[6] HART, J. E.-KUNEN, K.: On the regularity of the topology of separate continuity, Topology Appl. 123 (2002), 103-123.

[7] HENRIKSEN, M.-WOODS, R. G.: Separate versus joint continuity: a tale of four topologies, Topology Appl. 97 (1999), 175-205.

[8] HOLÁ, L.-PIOTROWSKI, Z.: Set of continuity points of functions with values in generalized metric spaces, Tatra Mt. Math. Publ. 42 (2009), 149-160.

[9] JUHÁSZ, I.: Cardinal Functions in Topology, in: Math. Centre Tracts 34, Amsterdam, 1975.

[10] KURATOWSKI, K.: Topology. Vol. I. PWN-Polish Scientific Publishers, Warszawa, 1966.

[11] PIOTROWSKI, Z.: Dense subsets of product spaces, Questions Answers Gen. Topology 11 (1993), 135-13.

[12] POPVASSILEV, S.: Non-regularity of some topologies on $\mathbb{R}^{n}$ stronger than the standard one, Math. Pannon. 5 (1994) 105-110.

[13] SCHRÖDER, J.: Impossible thin dense sets, Questions Answers Gen. Topology 13 (1995), 93-96.

[14] SIERPÍNSKI, W.: Sur une propriété de fonctions de deux variables réelles, continues par rapport à chacune de variables, Publ. Math. Univ. Belgrade 1 (1932) 125-128.

[15] STEEN, L. A.-SEEBACH, J. A.: Counterexamples in Topology. Holt, Rinehart and Winston, New York, 1970.

[16] SZEPTYCKI, P. J.: Dense subsets of product spaces, Questions Answers Gen. Topology 13 (1995), 221-222.

[17] VAN DOUWEN, E. K.: The Integers and Topology, in: Handbook of Set-Theoretic Topology (K. Kunen, J. E. Vaughan, eds.), North-Holland, Amsterdam, 1984. 


\title{
ON THE SEPARATELY OPEN TOPOLOGY
}

[18] VELlemAN, D. J.: Multivariable calculus and the plus topology, Amer. Math. Monthly 106 (1999), 733-740.

Received September 22, 2007

\author{
Zbigniew Piotrowski \\ Eric Wingler \\ Department of Mathematics \\ Youngstown State University \\ Youngstown, $\mathrm{OH} 44555$ \\ U.S.A. \\ E-mail: zpiotr@math.ysu.edu \\ wingler@math.ysu.edu \\ Robert W. Vallin \\ Department of Mathematics \\ Slippery Rock University of PA \\ Slippery Rock, PA 16057 \\ U.S.A. \\ E-mail: robert.vallin@sru.edu
}

